\title{
Evaluation of equine oocyte developmental competence using polarized light microscopy
}

\author{
A Bertero ${ }^{1}$, F Ritrovato ${ }^{2}$, F Evangelista ${ }^{2}$, V Stabile ${ }^{2}$, R Fortina $^{3}$, A Ricci ${ }^{1}$, A Revelli ${ }^{2}$, L Vincenti $^{1}$ \\ and $\mathrm{T} \mathrm{Nervo}^{1}$ \\ ${ }^{1}$ Department of Veterinary Science, University of Torino, Grugliasco, Italy, ${ }^{2}$ Department of Surgical Sciences, \\ University of Torino, S. Anna Hospital, Torino, Italy and ${ }^{3}$ Department of Agricultural, Forestry and Food Sciences, \\ University of Torino, Grugliasco, Italy \\ Correspondence should be addressed to A Bertero; Email: alessia.bertero@unito.it
}

\begin{abstract}
The purpose of this study was to observe in vitro-matured equine oocytes with an objective computerized technique that involves the use of a polarized light microscope (PLM) in addition to the subjective morphological evaluation obtained using a classic light microscope $(\mathrm{LM})$. Equine cumulus-oocyte complexes (COCs, $n=922)$ were subjected to different in vitro maturation times $(24,36$ or $45 \mathrm{~h}$ ), however, only 36-h matured oocytes were analyzed using CLM. The 36-h matured oocytes that reached maturity were parthenogenetically activated to evaluate the quality and meiotic competence. Average maturation percentages per session in groups 1,2 and 3 (24-, 36- and 45-h matured oocytes respectively) were $29.31 \pm 13.85,47.01 \pm 9.90$ and $36.62 \pm 5.28 \%$, whereas the average percentages of immature oocytes per session were $28.78 \pm 20.17,7.83 \pm 5.51$ and $22.36 \pm 8.39 \%$ respectively. The zona pellucida (ZP) birefringent properties were estimated and correlated with activation outcome. ZP thickness and retardance of the inner layer of the zona pellucida (IL-ZP) were significantly increased in immature oocytes compared with mature oocytes $(P<0.001$ and $\boldsymbol{P}<0.01$ respectively). The comparison between parthenogenetically activated and non-activated oocytes showed a significant increase in the area and thickness of the IL-ZP in parthenogenetically activated oocytes $(P<0.01)$. These results show that the 36 -h in vitro maturation (IVM) protocol allowed equine oocytes to reach maturity, and PLM observation of ZP can be used to distinguish mature and immature oocytes as well as activated and non-activated oocytes.

Reproduction (2017) 153 775-784
\end{abstract}

\section{Introduction}

In vitro maturation (IVM) of oocytes is a common technique used for years in assisted reproduction programs in veterinary medicine (Carneiro et al. 2002, Hinrichs 2011). It allows the successful maturation of oocytes recovered at any developmental stage by using specific culture media and conditions. During this process, gametes undergo structural and biological modifications before proceeding in their maturity (Carneiro et al. 2002).

There is a wide degree of variability in the maturation competence of equine oocytes (Hinrichs 2010), and only a restricted number of oocytes can reach the maturation stage suitable for subsequent fertilization. Identifying predictive markers of in vitro developmental competence could achieve higher performance percentages in assisted reproduction techniques. Analysis of cumulus oophorus, cytoplasmic and nuclear aspects, polar body presence, vital staining and parthenogenetic activation are suitable for these purposes (Carneiro et al. 2002, Hinrichs 2011, Koester et al. 2011, Yeste et al. 2016).
Some researchers have stated that some morphological parameters of oocytes and cumulus oophorus are useful elements for evaluating the quality of gamete, including the expansion and integrity grade of cumulus oophorus, the presence of polar bodies, the absence of zona pellucida (ZP) abnormalities and the absence of cytoplasmic fragments in the perivitelline space (Dell'Aquila et al. 1997a, Galli et al. 2007, Caamaño et al. 2010).

Other techniques require either fixation and/or fluorescence staining and confocal microscopy to analyze the spindle structure after immunostaining of chromatin and/or tubulin and electron microscopy techniques (Hinrichs et al. 1993, Tharasanit et al. 2006, Siddiqui et al. 2009). These methods have various disadvantages including their invasiveness that renders the oocytes unusable after the evaluation. Other methods such as brilliant cresyl blue staining are not useful for predicting equine oocyte competence prior to IVM (Pereira et al. 2014). Moreover, the static nature of classical microscopy images impairs the dynamic evaluation of oocyte behavior (Caamaño et al. 2010). 
Table 1 COC collection.

\begin{tabular}{|c|c|c|c|c|c|c|c|}
\hline Date & No. of ovaries & No. of follicles & No. of COCs & Foll/ov & COCs/foll & COCs/ov & $\operatorname{Rec}(\%)$ \\
\hline Total & 166 & 1331 & 922 & 8.02 & 0.69 & 5.55 & 69.27 \\
\hline Average & 6.68 & 47.54 & 32.93 & 7.90 & 0.69 & 5.42 & 68.82 \\
\hline S.D. & 2.76 & 16.61 & 12.81 & 3.05 & 0.10 & 2.61 & 10.31 \\
\hline
\end{tabular}

Average, average per individual work session; COCs/foll, ratio of COCs to follicles; COCs/ov, ratio of COCs to ovaries; Foll/ov, ratio of follicles to ovaries; No. of COCs, total no. of collected COCs; No. of follicles, total no. of dissected follicles; No. of ovaries, total no. of collected ovaries; Rec, collection percentage.

In this context, polarized light microscope (PLM), a technique normally used for the characterization of human oocytes in assisted fertilization treatments, offers dynamic evaluation of oocyte behavior and could be useful for equine assisted reproduction.

The purpose of this study was to observe in vitromatured equine oocytes with an objective computerized technique that involves the use of PLM in addition to the subjective morphological evaluation obtained using a classic bright field light microscope (LM).

The literature describes different maturation times for equine oocytes based on cumulus morphology (culture is performed for 24-30h for expanded oocytes and $30-36 \mathrm{~h}$ for compact oocytes). According to previous work (Hinrichs et al. 2005), the collected cumulusoocyte complexes (COCs) were classified based on cumulus morphology and subjected to 24-, 36- or 45-h IVM. As 36-h matured oocytes exhibit better maturity and viability, and due to facility constraints, only the 36-h oocytes were observed under PLM and LM to identify morphological signs of successful maturation.

\section{Materials and methods \\ Ovary collection and dissection}

The ovaries were collected over a 14-month period at a local abattoir immediately after evisceration of 104 mares with unknown reproductive history and were transported at $35-37^{\circ} \mathrm{C}$ in a sterile physiological saline solution (SPSS) to the laboratory within $4-5 \mathrm{~h}$ to ensure the best standardized conditions (Gambini et al. 2014). Immature or abnormal gonads were excluded from the study. On arrival, ovaries were dissected from the surrounding connective tissues, rinsed with a $10 \%$ Virkon solution and maintained in SPSS at a temperature of $35-37^{\circ} \mathrm{C}$.

A laminar flow hood, sterile surgical instruments and sterile gloves were used for follicle dissection. First, the tunica albuginea and interstitial tissues were removed to expose the follicular wall. Each follicle was sliced using a scalpel blade, and the antral surface of the follicular wall was scraped using a Volkmann spoon to ensure release of the COCs from the follicular wall. The remnants of the ovary (follicle remnants) were additionally washed with the dissection medium (DM) mentioned below, using a 20-mL syringe with a 21-G needle.

The follicular contents were collected in $50 \mathrm{~mL}$ Petri dishes and mixed with DM composed of M199 (15.06g/L) supplemented with penicillin $(0.07 \mathrm{~g} / \mathrm{L})$, streptomycin $(0.07 \mathrm{~g} / \mathrm{L})$, heparin $(0.01 \mathrm{~g} / \mathrm{L})$, bovine serum albumin (BSA, $1 \mathrm{~g} / \mathrm{L}), \mathrm{NaOH}(13.00 \mathrm{mM})$ and $\mathrm{NaHCO}_{3}(4.05 \mathrm{mM})$ at $38^{\circ} \mathrm{C}$ with a $\mathrm{pH}$ adjusted to 7.2-7.4. The medium was placed in a humidified incubator at $38.5^{\circ} \mathrm{C}$ with $5 \% \mathrm{CO}_{2}$ in air. The number of dissected follicles was recorded for each Petri dish to quantify the COC recovery rate (Table 1 ).

\section{COC collection and classification}

Culture dishes (GWst-3522, 50 $\times 7 \mathrm{~mm}$, WillCo-Wells, the Netherlands) were placed in a humidified incubator (THERMO Steri-cycle $\mathrm{CO}_{2}$ incubator HEPA Class 100 ) at $38.5^{\circ} \mathrm{C}, 5 \% \mathrm{CO}_{2}$ in air and subsequently examined using a stereomicroscope equipped with a warm stage (Wild Heerbrugg-M32) at 6-45× magnification. To improve microscopic observation in the presence of debris, the contents of a single dish were divided into additional Petri dishes, diluting the contents with more medium. Once identified, the COCs were collected using a glass capillary with a mouth pipette and transferred to a 35-mm Petri dish filled with DM.

The recovered COCs were divided into three categories based on their morphology: Group 1 (oocytes with a compact multilayered cumulus), Group 2 (oocytes with an expanded cumulus) and Group 3 (oocytes with only the corona radiata) (Fig. 1). The three COC categories were kept separate during the following experiments. Some authors have reported maturation rates of $51 \%$ and $60 \%$ in compact and expanded cumulus COCs respectively (Galli et al. 2007). The morphological criterion used for COC evaluation was based on previous publications (Hinrichs \& Williams 1997, Tharasanit et al. 2006, Hinrichs 2011). COCs were classified
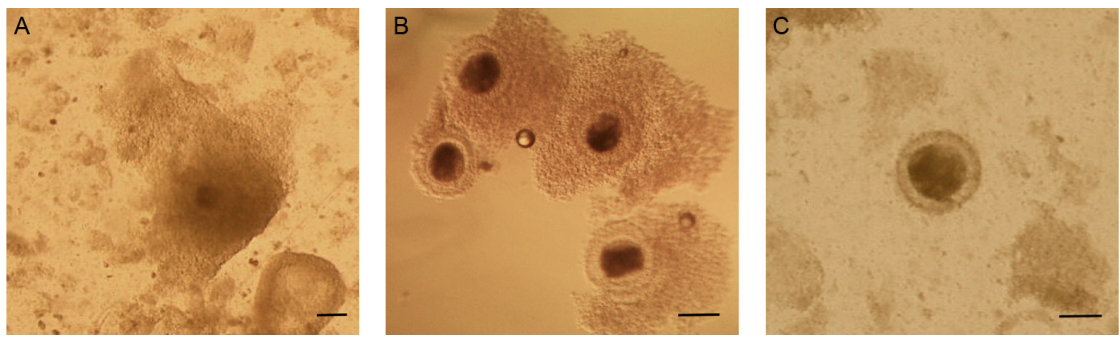

Figure 1 Compact $(\mathrm{A})$, expanded $(\mathrm{B})$ and corona radiata (C) COCs. Bar $=100 \mu \mathrm{m}$. 
as compact when the granulosa appeared as sheets of tissue with an even surface that may be thrown into folds by the scraping with a curette. This tissue, as seen on the surface of a fold or on the hillock covering the oocyte, was smooth without evidence of protruding cells. The compact COC was typically shaped like a flat-brimmed hat, with a flat underside and a bulging upper surface covered by a 'hillock' of cumulus. Only those COCs with both compact cumuli and compact mural granulosa cells were classified as compact. In expanded COCs, the granulosa cells were not in smooth sheets or flat layers. This includes some cells protruding from a sheet, sheets with a thickened or puffy granular appearance and clouds of expanded cells. Very expanded granulosa could be associated with a jelly-like yellow substance. Compact granulosa originates from viable follicles, whereas expanded granulosa originates from atretic follicles (Hinrichs \& Williams 1997). In other species, oocytes with expanded granulosa at the time of collection are discarded as degenerated. However, both types of horse oocyte are valuable (Hinrichs \& Williams 1997, Hinrichs 2011) (Fig. 1).

The COCs were exposed to mechanical trauma during collection and washing, and partial or total loss of the cumulus can occur. This condition was observed in COCs with an incomplete cumulus or only the corona radiata.

The oocytes with a diaphanous aspect and prominent nucleus or with evident abnormalities (poor and inhomogeneous cytoplasm, fragmented and pyknotic nucleus) were excluded from the experiment because of non-viability.

\section{In vitro maturation}

The COCs considered morphologically suitable for IVM were transferred to four-well dishes filled with 500- $\mu \mathrm{L}$ maturation medium (MM). Approximately, 10 COCs were placed in each well. The category division (compact, expanded and corona radiata) was maintained to evaluate the difference in IVM response between COC categories. The MM used was M199 $(9.70 \mathrm{~g} / \mathrm{L})$ modified with the addition of fetal calf serum (FCS, 10\%), epidermal growth factor (EGF, $0.0005 \mathrm{~g} / \mathrm{L})$, lactate $(66.04 \mathrm{mM})$, cystine $(0.37 \mathrm{mM})$, cysteamine $(0.52 \mathrm{mM})$, myo-inositol $(0.03 \mathrm{mM})$, ascorbic acid $(0.40 \mathrm{mM})$, glutamine $(0.62 \mathrm{mM})$, sodium pyruvate $(0.91 \mathrm{mM})$, insulin-transferrinsodium selenite (ITS, $0.001 \mathrm{~g} / \mathrm{L}$ ) and menotropin (Menogon, Ferring S.p.a, 46.95 IU/L) and adjusted to $\mathrm{pH}$ 7.2. The complete process was performed within $5 \mathrm{~h}$ at a room temperature that ranged between $22^{\circ} \mathrm{C}$ and $26^{\circ} \mathrm{C}$ to prevent the impairment of vitality and maturation competence (Pedersen et al. 2004).

Homogeneous groups of the three categories of COCs, consisting of approximately the same number or proportion of compact, expanded and corona radiata COCs, were assigned to different incubation times $(24,36$ or $45 \mathrm{~h})$ and placed in the incubator at $38.5^{\circ} \mathrm{C}$ with $5 \% \mathrm{CO}_{2}$ in air. The purpose was to determine if 36 -h maturation time under our laboratory conditions was suitable to obtain adequate maturation percentages because only $36-\mathrm{h}$ matured COCs were submitted for PLM observation. The COCs incubated for 24 and $45 \mathrm{~h}$ were only submitted to morphological examination by stereomicroscope at $60 \times$ magnification (for evaluation of cumulus morphology, cytoplasm segregation of darkness and light zones (Hinrichs 2011), presence of polar body), and the results were compared with 36-h matured COCs.

\section{Oocyte decumulation}

According to previously described criteria, the COCs were evaluated at the end of the assigned maturation period for morphologic assessment of the cumulus (expanded or still compact) (Hinrichs \& Williams 1997, Alm \& Hinrichs 2000, Hinrichs \& Schmidt 2000). After COC observation, the cumulus was removed. Decumulation is a necessary step for microscopic evaluation of the polar body and the nucleus and for subsequent PLM analysis of oocytes. Enzymatic digestion with a hydrolyzing solution combined with mechanical aspiration into decreasing diameters of glass capillaries was used to fragment the junctional desmosome of the cumulus. The procedure was repeated four times in four different 35-mm Petri dishes maintained at a temperature of $38.5^{\circ} \mathrm{C}$ and filled with $2 \mathrm{~mL}$ of four different types of media: (1) HEPES synthetic oviductal fluid (H-SOF) (Tremoleda et al. 2003) supplemented with $20 \mu \mathrm{L}$ hyaluronidase $(0.0025 \mathrm{~g} / \mathrm{L})$; $(2)$ trypsin $(2.50 \mathrm{~g} / \mathrm{L})$ buffered with HEPES (19.97 mM) and supplemented with EDTA $(1.37 \mathrm{mM})$, D-glucose (5.55 mM), NaCl (119.78 mM), Na $\mathrm{HPO}_{4}(0.84 \mathrm{mM})$, $\mathrm{KH}_{2} \mathrm{PO}_{4}(1.76 \mathrm{mM})$ and $\mathrm{KCl}(4.9 \mathrm{mM})$; (3) H-SOF supplemented with $200 \mu \mathrm{L}$ FCS and (4) H-SOF. Non-essential MEM $(10 \mathrm{~mL} / \mathrm{L})$ and essential MEM (20 mL/L) buffered with HEPES (19.97 mM) and supplemented with $\mathrm{CaCl}_{2} .2 \mathrm{H}_{2} \mathrm{O}(1.70 \mathrm{mM}), \mathrm{MgCl}_{2} .6 \mathrm{H}_{2} \mathrm{O}$ (0.49 mM), $\mathrm{NaCl}$ (115.84 mM), $\mathrm{KCl}$ (4.02 mM), $\mathrm{KH}_{2} \mathrm{PO}_{4}$ $(1.18 \mathrm{mM}), \mathrm{NaHCO}_{3}(5.00 \mathrm{mM})$, sodium pyruvate $(0.33 \mathrm{mM})$, penicillin $(0.063 \mathrm{~g} / \mathrm{L})$, streptomycin $(0.050 \mathrm{~g} / \mathrm{L})$, sodium lactate $(6.60 \mathrm{mM})$, D-glucose $(1.50 \mathrm{mM}), \mathrm{BSA}$ fraction $\mathrm{V}(4 \mathrm{~g} / \mathrm{L})$, glycine $(9.99 \mathrm{mM})$ and alanyl glutamine $(1.01 \mathrm{mM})$ were used to obtain $\mathrm{H}$-synthetic oviductal fluid (H-SOF) medium. All the reagents were purchased from Sigma-Aldrich.

First, the COCs were transferred by gentle aspiration into a glass capillary to stimulate cumulus cell removal by mechanical action from the MM into the hyaluronidase-supplemented medium (medium 1) to ensure standardized exposure to the enzymatic medium and obtain the first digestion of cell junctions. The oocytes were then placed into the trypsin solution (medium 2) for no longer than 90 s to further reduce cohesion between cumulus and corona radiata cells. To ease the final denudation, COCs were maintained in medium 3 and aspirated into progressively decreasing diameter capillaries to allow the mechanical detachment of the corona radiata cells from the ZP. In the last Petri dish (medium 4), the oocytes were rinsed for approximately $1 \mathrm{~min}$ to remove the remaining cellular debris and any traces of enzymatic solutions.

The oocytes to be submitted to PLM analysis were selected at the end of the IVM period on the basis of morphological characteristics. The oocytes that showed signs of degeneration (e.g., the presence of ZP anomalies and/or the presence of cytoplasmic fragments in the perivitelline space or pyknotic nucleus) were excluded from the study. The selected oocytes $(n=127)$ grouped with respect to the initial cumulus morphology were placed in Eppendorf tubes with $300 \mu \mathrm{L}$ TCM-199 supplemented with gentamicin $(0.05 \mathrm{~g} / \mathrm{L})$, sodium pyruvate $(0.20 \mathrm{mM}), \mathrm{NaHCO}_{3}(4.17 \mathrm{mM})$ and $10 \% \mathrm{FCS}$ at $38.5^{\circ} \mathrm{C}$ and transported in an insulated flask to the FIVER laboratory of Sant'Anna Hospital, University of Turin, Italy. 


\section{Polarized light microscope analysis}

For the non-invasive evaluation of oocyte quality, PLM analysis was carried out using a polarized light microscope (PolScope) equipped with Oosight software to automatically acquire the ZP birefringence measurements. The PLM technique allows detailed visualization of the ZP structure and identification of three ZP layers (inner, IL; outer, OL and middle layer, ML) (Keefe et al. 1997, Pellettier et al. 2004, Koester et al. 2011). In accordance with the study by Mohammadi-Sangcheshmeh and coworkers (Mohammadi-Sangcheshmeh et al. 2014), our investigation confirmed the presence of the three ZP layers in equine oocytes and our study specifically analyzed the IL-ZP, which is characterized by greater birefringence.

Maintaining the initial cumulus morphology grouping, the following parameters were considered: (i) total area and thickness of the IL-ZP; (ii) retardance of the IL-ZP and (iii) total ZP thickness. Retardance is considered as an expression of structural density; the highest retardance values correspond to the highest density values. The observations were performed with an inverted microscope (Nikon Eclipse TE 2000-S) equipped with a LC PolScope controller (CRI, USA), a colorcoded Doppler (CCD) camera and a heated ceramic plate. The images were acquired at $40 \times$ magnification, and Oosight software was used to obtain the measurements. The oocytes were placed in $8 \mu \mathrm{L}$ drops of equilibrated HEPES-buffered medium (Gamete Medium, Cook, Ireland) covered with $3 \mathrm{~mL}$ equilibrated paraffin oil (Oil Medium, Cook, Ireland) in glass Petri dishes (GWst-3522, 50×7 mm, WillCo-Wells). To achieve optimal visualization of the anisotropic structures during the examination, oocytes were rotated using an injection pipette (Injection Pipette, Cook, Ireland) to place the polar body and ZP in the same focal plane. To measure ZP thickness, a line between the innermost (contiguous with the oolemma) and outermost point of the ZP was drawn. The measurements were taken twice per each sample to obtain average records, and the images were collected. Due to the heterogeneous characteristics of the ZP, the measurements expressed in micrometers were taken at four different points to ensure accurate assessment, and the mean of these four values was used for the statistical analysis (Kilani et al. 2006).

\section{Parthenogenetic activation}

In accordance with the literature, to demonstrate the final viability of oocytes matured for $36 \mathrm{~h}$ and after PLM evaluation (Fernandes et al. 2014), 167 oocytes were submitted to parthenogenetic activation using ionomycin- and 6-dimethylaminopurine (6-DMAP)-supplemented medium as activating agents (Liu et al. 1998). First, the oocytes were transferred for $5 \mathrm{~min}$ into 35 - $\mathrm{mm}$ Petri dishes with $5 \mathrm{~mL} \mathrm{TCM}$ 199 with $10 \%$ FCS added with $10 \mu \mathrm{L}$ ionomycin $(2.48 \mathrm{mM})$. Then, the gametes were washed several times in TCM-199 supplemented with $10 \%$ FCS and incubated in another $35-\mathrm{mm}$ Petri dish with $5 \mathrm{~mL} \mathrm{TCM}-199$ with $10 \%$ FCS and $2 \mathrm{mg}$ 6-DMAP $(2.45 \mathrm{mM})$ for $5 \mathrm{~h}$ at $38.5^{\circ} \mathrm{C}$ under $5 \% \mathrm{CO}_{2}$ in air.

At the end of the incubation, the oocytes underwent serial washes in TCM-199 supplemented with $10 \%$ FCS. The cells were then placed in $10-\mu \mathrm{L}$ drops of SOF
(Tremoleda et al. 2003), covered with equilibrated paraffin oil in 35-mm glass Petri dishes and incubated in a jar at $38.5^{\circ} \mathrm{C}$ with $5 \% \mathrm{O}_{2}$ and $5 \% \mathrm{CO}_{2}$ for $2-6$ days. To assess the activation progress, dishes were examined daily to verify achievement of the first cleavage due to the first mitotic cell cycle and cleavage progression timing. Non-essential MEM (10 mL/L) and essential MEM (20 mL/L): $\mathrm{CaCl}_{2} \cdot 2 \mathrm{H}_{2} \mathrm{O}$ (1.70 mM), $\mathrm{MgCl}_{2} .6 \mathrm{H}_{2} \mathrm{O}(0.49 \mathrm{mM}), \mathrm{NaCl}(92.40 \mathrm{mM}), \mathrm{KCl}$ (7.11 mM), $\mathrm{KH}_{2} \mathrm{PO}_{4}$ (1.18 mM), $\mathrm{NaHCO}_{3}$ (26.19 mM), HEPES $(19.97 \mathrm{mM})$, Na-pyruvate $(0.33 \mathrm{mM})$, penicillin $(0.063 \mathrm{~g} / \mathrm{L})$, streptomycin $(0.050 \mathrm{~g} / \mathrm{L})$, Na-lactate $(3.30 \mathrm{mM})$, D-glucose $(1.50 \mathrm{mM})$, bovine serum albumin fatty acid free (BSA FAF, $4 \mathrm{~g} / \mathrm{L})$, glycine $(9.99 \mathrm{mM})$ and alanyl glutamine $(1.01 \mathrm{mM})$ were used to obtain the SOF medium.

\section{Statistical analysis}

The results were expressed as mean \pm S.D. and were graphically represented in histograms. Statistical analysis was performed using GraphPad InStat (GraphPad Software). Student's $t$ tests were used to compare two sets of quantitative data when samples were collected independently from one another (for unpaired samples), and non-parametric Mann-Whitney $U$ tests were used to compare non-Gaussian variables.

Analysis of variance (ANOVA) for parametric data and Kruskal-Wallis tests for non-parametric data followed by an appropriate post hoc test (Bonferroni or Dunn's tests respectively) was used to compare more than two data sets. To choose between parametric or non-parametric tests, sample distributions were evaluated using the Kolmogorov-Smirnov test. In all of the analyses, differences were considered statistically significant when $P<0.05$.

\section{Results \\ COC collection and IVM}

A total of 187 ovaries were collected from 104 mares. Ten ovaries were abnormal and subsequently excluded from the study, and an additional 11 ovaries were excluded as they did not yield any follicles. Thus, the study used 166 ovaries from which 1331 follicles were dissected (scraped), with an average of 8.02 follicles per ovary.

This yielded a total of 922 COCs of which $50.00 \%$ $(n=461)$ were scored as expanded COCs, $35.79 \%$ $(n=330)$ were compact, $10.41 \%(n=96)$ had only the corona radiata and $3.80 \%(n=35)$ were degenerated or lacked the ZP (Fig. 1). Of these, only 805 COCs that were a mix of expanded, compact and corona radiata COC types were selected for the study.

Thus, among all COCs, 805 (87.31\%) were selected for IVM and 117 were eliminated because of their incomplete/irregular cumulus. These 805 COCs included in this study were processed during 24 work sessions. The COCs were classified and grouped based on cumulus characteristics: 300 compact COCs (37.27\%), 
Table 2 Maturation outcome assessed after $24 \mathrm{~h}$ of IVM culture.

\begin{tabular}{lcccrrr}
\hline & No. of COCs & CYT mat $(\%)$ & PB mat $(\%)$ & CYT + PB mat $(\%)$ & \multicolumn{1}{c}{ IMM $(\%)$} & DEG $(\%)$ \\
\hline Total & 71 & $7(9.86)$ & $15(21.13)$ & $22(30.99)$ & $25(35.21)$ & $24(33.80)$ \\
Average & 17.75 & $1.75(13.21)$ & $3.75(16.09)$ & $5.50(29.31)$ & $6.25(28.78)$ & $6.00(41.91)$ \\
S.D. & 8.18 & $2.87(20.32)$ & $4.50(18.61)$ & $3.32(13.85)$ & $7.23(20.17)$ & $2.16(24.45)$ \\
\hline
\end{tabular}

Unless indicated otherwise, data show the number of oocytes in each group, with percentages given in parentheses.

Average, average per individual work session; CYT mat, oocytes showing signs of cytoplasmic maturation; CYT + PB mat, total value of CYT mat+PB mat; DEG, degenerate oocytes; IMM, immature oocytes; PB mat, oocytes presenting polar body extrusion.

421 expanded COCs (52.30\%) and $84(10.43 \%)$ COCs showing only the corona radiata.

\section{In vitro maturation}

\section{Maturation output of the three groups}

The groups submitted to three different maturation times $(24,36$ or $45 \mathrm{~h})$ were as follows: 71 COCs submitted to the 24-h maturation protocol (29 compact cumulus, 40.84\%; 33 expanded cumulus, $46.48 \%$; and 9 corona radiata, $12.68 \%), 537$ COCs submitted to the $36-\mathrm{h}$ maturation protocol (203 compact cumulus, $37.80 \%$; 283 expanded cumulus, $52.70 \%$; and 51 corona radiata, $9.50 \%$ ) and 197 COCs submitted to the 45 -h maturation protocol (68 compact cumulus, 34.52\%; 105 expanded cumulus, $53.30 \%$; and 24 corona radiata, $12.18 \%$ ). To verify the maturation rate, after decumulation that took place at the end of each maturation time $(24,36$ or $45 \mathrm{~h})$, all the oocytes were observed at $60 \times$ magnification using a stereomicroscope equipped with a warm stage (Wild Heerbrugg-M32). In Group 1 (24 h), 22 (30.99\%) mature oocytes were detected, (i.e. oocytes showing both cytoplasmic (cytoplasm segregation of darkness and light zones) and nuclear maturity (presence of polar body extrusion as a consequence)), with an average of $5.5 \pm 3.32$ oocytes per maturation session. In Group 2 (36-h incubation), 253 (47.11\%) mature oocytes were observed with an average of $16.87 \pm 6.23$ oocytes per maturation session. In Group 3 (45-h incubation), 73 $(37.06 \%)$ mature oocytes with an average of $14.60 \pm 4.34$ oocytes per maturation session were detected. The average percentages of maturation per session in groups 1, 2 and 3 were $29.31 \pm 13.85,47.01 \pm 9.90$ and $36.62 \pm 5.28 \%$ respectively. Finally, the average percentages of immature oocytes per work session were $28.78 \pm 20.17, \quad 7.83 \pm 5.51$ and $22.36 \pm 8.39 \%$ in Group 1 (Table 2), Group 2 (Table 3) and Group 3 (Table 4) respectively.

\section{Comparison between IVM incubation times}

We statistically compared the average maturation percentage and average percentage of oocytes that failed to mature. Data were grouped by single work session and related to the different IVM incubation times. In our laboratory conditions, the IVM protocol with 36-h incubation (Group 2) showed the best output in terms of maturation percentage per single session. Specifically, the differences between the percentages obtained from Groups 1 and 2 were significant $(P<0.05)$. Regarding the average percentages of immature oocytes per single work session, the differences between values obtained from Group 2 and the other two groups were significant $(P<0.05)$.

\section{PLM analysis}

\section{Mature and immature oocytes analysis}

A group of 127 oocytes randomly selected from the 537 oocytes subjected to 36-h IVM was analyzed using PLM. Of these oocytes, 36 were immature and 91 were mature with evident polar body extrusion (Fig. 2). Based on ZP birefringence parameters, the oocytes were subdivided into mature or immature, and the results were compared (Figs 3, 4, 5 and 6). The average retardance showed a significant increase $(P<0.01)$ in immature oocytes $(2.71 \pm 1.06 \mathrm{~nm})$ compared with that for mature oocytes $(2.12 \pm 0.54 \mathrm{~nm})$. Similarly, the average ZP thickness was significantly increased $(P<0.001)$ in immature oocytes $(20.07 \pm 2.93 \mu \mathrm{m})$ compared with that of mature oocytes $(17.90 \pm 2.53 \mu \mathrm{m})$. No statistically significant differences were recorded between the two groups in terms of the area or thickness of the IL-ZP.

\section{Activation}

Quality and meiotic competence of the oocytes after IVM were evaluated based on parthenogenetic activation of

Table 3 Maturation outcome assessed after $36 \mathrm{~h}$ of IVM culture.

\begin{tabular}{lccrrrr}
\hline & No. of COCs & CYT mat $(\%)$ & PB mat $(\%)$ & \multicolumn{1}{c}{ CYT + PB mat $(\%)$} & IMM $(\%)$ & DEG $(\%)$ \\
\hline Total & 537 & $9(1.68)$ & $244(45.44)$ & $253(47.11)$ & $40(7.45)$ & $244(45.44)$ \\
Average & 35.80 & $0.60(1.67)$ & $16.27(45.34)$ & $16.87(47.01)$ & $2.67(7.83)$ & $16.27(45.16)$ \\
S.D. & 10.64 & $1.59(4.76)$ & $6.53(12.14)$ & $6.23(9.90)$ & $1.76(5.51)$ & $5.99(10.54)$ \\
\hline
\end{tabular}

Unless indicated otherwise, data show the number of oocytes in each group, with percentages given in parentheses.

Average, average per individual work session; CYT mat, oocytes showing signs of cytoplasmic maturation; CYT + PB mat, total value of CYT mat+ PB mat; DEG, degenerate oocytes; IMM, immature oocytes; PB mat, oocytes presenting polar body extrusion. 
Table 4 Maturation outcome assessed after $45 \mathrm{~h}$ of IVM culture.

\begin{tabular}{|c|c|c|c|c|c|c|}
\hline & No. of COCs & CYT mat $(\%)$ & PB mat (\%) & CYT + PB mat (\%) & IMM (\%) & DEG $(\%)$ \\
\hline Total & 197 & $19(9.64)$ & $54(27.41)$ & $73(37.06)$ & $42(21.32)$ & $82(41.62)$ \\
\hline Average & 39.40 & $3.80(9.29)$ & $10.80(27.33)$ & $14.60(36.62)$ & $8.40(22.36)$ & $16.40(41.02)$ \\
\hline S.D. & 8.96 & $3.49(7.45)$ & $5.17(11.16)$ & $4.34(5.28)$ & $2.19(8.39)$ & $6.02(9.28)$ \\
\hline
\end{tabular}

Unless indicated otherwise, data show the number of oocytes in each group, with percentages given in parentheses.

Average, average per individual work session; CYT mat, oocytes showing signs of cytoplasmic maturation; CYT + PB mat, total value of CYT mat+ PB mat; DEG, degenerate oocytes; IMM, immature oocytes; PB mat, oocytes presenting polar body extrusion.

the oocytes subjected to 36-h maturation, which were previously analyzed using the PolScope (Carneiro et al. 2001, Onigeri et al. 2001, Caamaño et al. 2010).

A total of 167 metaphase II oocytes (91 PLM observed and 76 randomly selected), derived from 70 compact COCs (41.92\%), 79 expanded COCs $(47.30 \%)$ and 18 corona radiata COCs $(10.78 \%)$ were parthenogenetically activated. Eighty-nine oocytes showed signs of activation, with an average of $57.28 \pm 22.62 \%$ (range, $17.39-85.71 \%$ ) activated COCs per session. The average activation percentages among different COC populations were evaluated every $24 \mathrm{~h}$ per single session as described previously and confirmed as positive when two-cell division was observed. Expanded COCs showed a greater activation percentage $(61.83 \pm 13.34 \%)$ compared with corona radiata $(27.08 \pm 29.46 \%)$ and compact COCs (57.53 $\pm 34.85 \%)$. Comparing the expanded and corona radiata oocytes, the difference between average activation percentages per single session was significantly $(P<0.05)$ in favor of expanded oocytes, which revealed good reproducibility per session.

\section{Differences in successfully and unsuccessfully activated oocytes observed by PLM}

Immediately after PLM analysis, 91 oocytes subjected to IVM for $36 \mathrm{~h}$ and observed by PLM underwent parthenogenetic activation. The percentage of oocytes that showed embryonic cleavage (activation rate) was $67.03 \% \quad(n=61)$. The values obtained from PLM examination (Fig. 7) of activated and non-activated oocytes were compared and statistically analyzed, and the results were represented graphically (Figs 3, 4, 5 and 6). The difference between the average IL-ZP area of activated oocytes $\left(2394.23 \pm 469.82 \mu \mathrm{m}^{2}\right)$ and that of non-activated oocytes $\left(2075.81 \pm 291.62 \mu \mathrm{m}^{2}\right)$ was
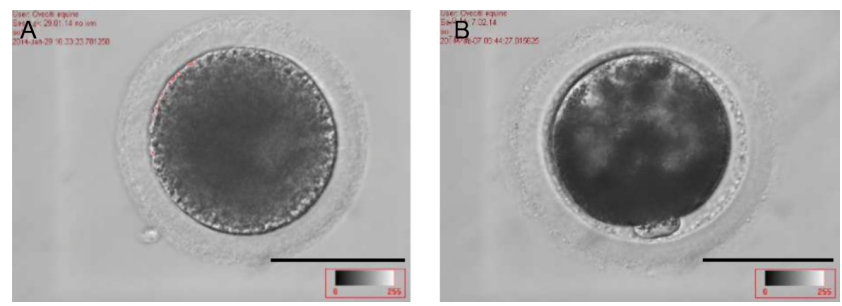

Figure 2 Immature oocyte (A) and oocyte showing signs of cytoplasmic and nuclear maturation (B). Bar $=100 \mu \mathrm{m}$. significant $(P<0.001)$. The average IL-ZP thickness of activated and non-activated cells was $4.68 \pm 1.07$ and $3.89 \pm 0.72 \mu \mathrm{m}$ respectively, $(P<0.01)$. However, the IL-ZP retardance and ZP thickness were not significantly different between the two groups of oocytes. The average retardance measurement among activated oocytes was $2.18 \pm 0.55 \mathrm{~nm}$, and the average ZP thickness was $18.14 \pm 2.37 \mu \mathrm{m}$, whereas the average retardance and ZP thickness measured among non-activated oocytes were $2.00 \pm 0.48 \mathrm{~nm}$ and $17.40 \pm 2.79 \mu \mathrm{m}$ respectively.

\section{Discussion}

PLM offers the advantage of being a non-invasive assessment; it is able to preserve oocyte viability and allows repeated observations of a sample. The PLM has the capability to emphasize and measure the molecular order of birefringent objects. The molecular order of the body is quantified by measuring the change in phase between the two polarized waves emerging from the object, and this parameter is called retardance. This and other ZP parameters linked to its structure are correlated with gamete fertility and with the ability of the oocyte to have better development after IVF (Shen et al. 2005, Rama Raju et al. 2007, Madaschi et al. 2009, Caamaño et al. 2010, Koester et al. 2011). The majority of studies using PLM have been performed on human, mice and bovine oocytes (Rama Raju et al. 2007, Caamaño et al. 2010, Koester et al. 2011). So far,

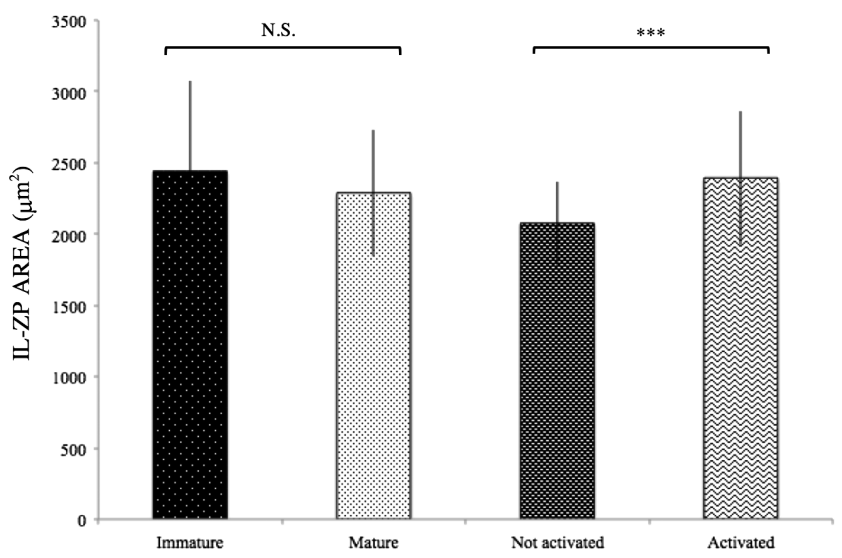

Figure 3 Graphical representation of the IL-ZP area values in immature/mature and non-activated/activated oocytes. ${ }^{* * *} P<0.001$; N.S., not significant. 


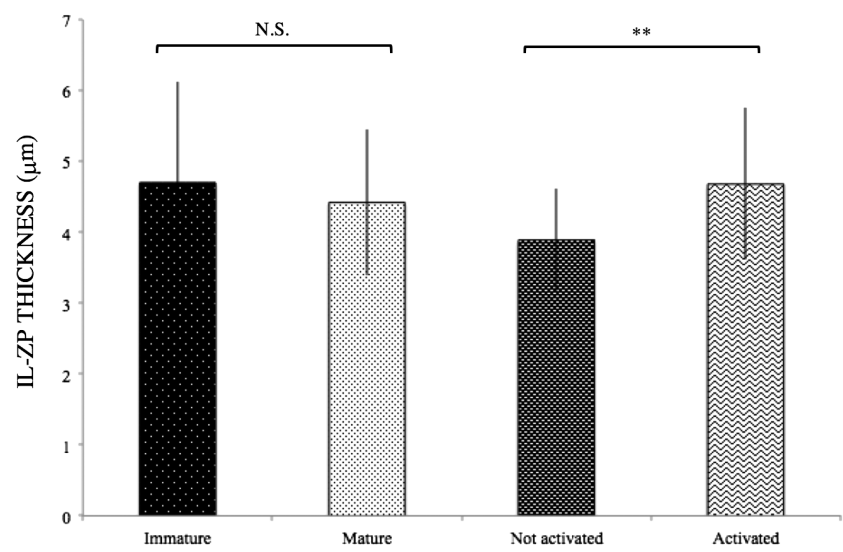

Figure 4 Graphical representation of the IL-ZP thickness in immature/mature and non-activated/activated oocytes. ${ }^{* *} P<0.01$ N.S., not significant.

only one study regarding the PolScopic evaluation of equine oocytes has been performed (MohammadiSangcheshmeh et al. 2014). The literature presents conflicting data regarding the correct incubation time required to induce complete IVM of equine oocytes. Wills and coworkers (Wills et al. 1991) stated that in vitro culture for either 15 or $32 \mathrm{~h}$ significantly increased the percentage of MII oocytes as compared with $0 \mathrm{~h}$ of culture and that $15 \mathrm{~h}$ of culture was sufficient time for equine oocyte IVM. Hinrichs and Schmidt (2000) and Galli and coworkers (Galli et al. 2007) used a 24-h IVM incubation time, whereas Mohammadi-Sangcheshmeh and coworkers (Mohammadi-Sangcheshmeh et al. 2014) used a IVM incubation time of 28-30 h. In our study, the $36-h$ incubation period was identified as the adequate culture time to obtain the highest number of oocytes characterized by full cytoplasmic and nuclear maturity (morphologically evaluated) and the lowest number of immature oocytes, compared with the 24- and 45-h incubation times. The percentages of cells matured

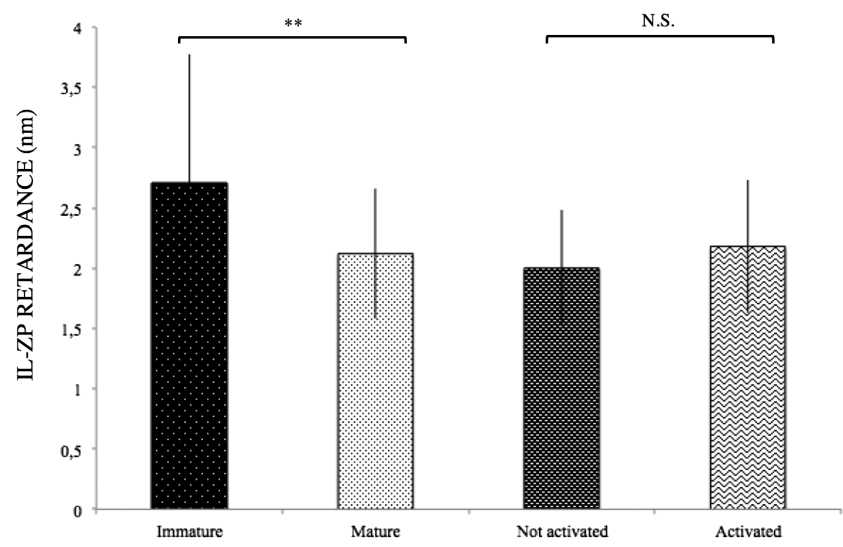

Figure 5 Graphical representation of the IL-ZP retardance in immature/mature and non-activated/activated oocytes. ${ }^{*} * P<0.01$; N.S., not significant.

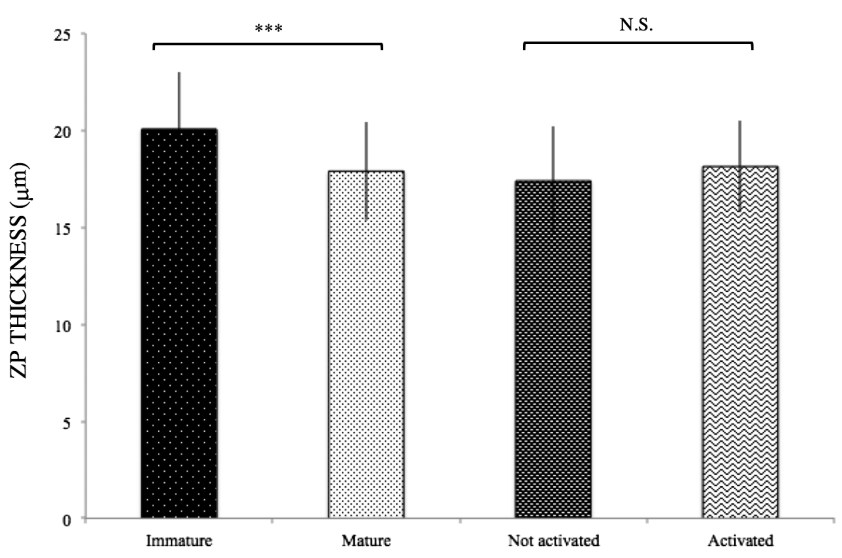

Figure 6 Graphical representation of the ZP thickness in immature/mature and non-activated/activated oocytes. ${ }^{* * *} P<0.001$; N.S., not significant.

in vitro using the 36 -h protocol were higher than the percentages achieved using the 24-h incubation time (47.11\% vs 30.99\%, $P<0.05)$. These results were similar to the values obtained by other groups (Willis et al. 1991, Dell'Aquila et al. 1997a, Hinrichs \& Schmidt 2000, Galli \& Lazzari 2001, Galli et al. 2002, 2007, Lagutina et al. 2005). Oocytes matured for $36 \mathrm{~h}$, presenting full cytoplasmic and nuclear maturation (with polar body extrusion), were subjected to parthenogenetic activation immediately after PLM analysis, which confirmed that oocyte quality and meiotic competence were adequate after $36 \mathrm{~h}$ of IVM. These results are also correlated with the birefringence analysis of the ZP.

We analyzed the correlation between oocyte developmental potential (in terms of percentage of oocytes that achieved in vitro activation) and the initial light microscopic morphological classification of 36-h matured oocytes. In our study, an average percentage of cleaved oocytes per session of $57.28 \pm 22.62 \%$ was obtained. This value was significantly higher than percentages reported in published literature for equine oocytes (28.5\%) (Carneiro et al. 2001). Among the analyzed oocytes, the average activation percentage per session of the expanded oocyte group $(61.83 \pm 13.34 \%)$ was higher than those of the compact group $(57.53 \pm 34.85 \%)$ and the corona radiata
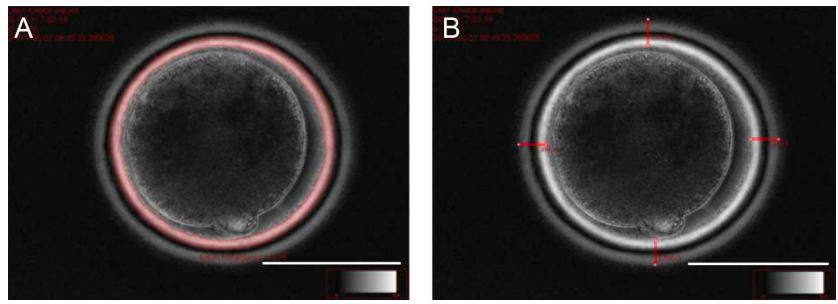

Figure 7 PLM analysis of the zona pellucida: measurement of the area and retardance of the ZP $(\mathrm{A})$ and measurement of the ZP thickness (B). Bar $=100 \mu \mathrm{m}$. 
group $(27.08 \pm 29.46 \%)$. The differences between the activation percentages of the corona radiata and expanded oocytes were significant $(P<0.05)$. These data are in accordance with the results obtained in the study by Mohammadi-Sangcheshmeh and coworkers in 2014 after intracytoplasmic sperm injection (ICSI). They obtained $50.7 \%$ cleaved oocytes among expanded COCs, even though this result was not statistically significant. The oocytes derived from expanded COCs and matured for $36 \mathrm{~h}$ showed the best ability to be stimulated by parthenogenetic activation. Several works have reported that developmental competence differs between expanded and compact equine oocytes (Hinrichs et al. 1995, 2002, Dell'Aquila et al. 1997b, Hinrichs \& Williams 1997, Franz et al. 2003, Choi et al. $2004 a, b)$. Nevertheless, these differences have not been completely characterized or understood because of the complexity of the events, and the large number of involved factors, such as maturation conditions and embryo culture media composition (Choi et al. $2004 a, b)$. Therefore, it cannot be unequivocally stated that expanded oocytes are best suited for fertilization/ activation procedures.

We also analyzed the correlation between oocyte developmental potential, ZP birefringence, IL-ZP area and thickness and total ZP thickness. This allowed us to compare the subjective morphological evaluation obtained using CLM on 36-h mature and immature oocytes with objective computerized evaluations obtained with PLM. The retardance of the IL-ZP and total thickness of the ZP were significantly increased in immature oocytes compared with that of mature oocytes. These findings are in accordance with previously reported bovine oocyte data (Koester et al. 2011, Held et al. 2012) that revealed that more advanced maturation stages correspond to lower birefringence values of the $\mathrm{ZP}$, contrary to what was observed in human oocytes (Shen et al. 2005, Rama Raju et al. 2007). Our results are in disagreement with other studies in which expanded oocytes with high developmental capacity, determined by cumulus morphology, had a significantly thicker and higher ZP retardance compared with compact oocytes (Mohammadi-Sangcheshmeh et al. 2014). Moreover, that group found that oocytes characterized by higher developmental competence (assessed by the evaluation of the glucose-6-phosphate dehydrogenase activity with brilliant cresyl blue testing) had a significantly thicker ZP and higher ZP birefringence.

Parthenogenetic activation also allowed us to assess whether the parameters measured after IVM using PLM were different in activated oocytes compared to non-activated oocytes and thus to correlate the activation ability with these parameters. This evaluation revealed that both the area and thickness of the IL-ZP were significantly increased in oocytes that responded positively to the activation procedure. These values could thus be considered predictive of achieving an adequate maturation status and good meiotic competence for oocytes after IVM. However, no statistically significant differences were found in IL-ZP retardance or total ZP thickness between the two groups, although we noticed a tendency toward increased values in activated oocytes.

In human medicine, there are many conflicting reports regarding thickness and area of the inner layer of the ZP. In a previous work, the IL-ZP area of both oocytes and embryos was significantly lower in conception than that in non-conception cycles, whereas IL-ZP retardance values of oocytes and embryos were comparable in conception and non-conception cycles (Molinari et al. 2012). In contrast, Shen and coworkers (Shen et al. 2005) demonstrated that IL-ZP thickness was slightly elevated and mean IL-ZP retardance was higher in oocytes contributing to conception cycles compared to non-conception cycles. Nevertheless, the ZP and spindle morphology measured by PLM can be considered a prognostic marker for oocyte quality (Wang et al. 2001a,b, Cooke etal. 2003, Keefe et al. 2003, Moon et al. 2003, Cohen et al. 2004, Pellettier et al. 2004, Rienzi et al. 2004, Trimarchi et al. 2004, Rama Raju et al. 2007, Ebner et al. 2008, Montag \& van der Ven 2008, Madaschi et al. 2009). Specifically, the inner layer of the ZP is crucial for embryonic development, and higher IL-ZP thickness and retardance values are related to greater potential for oocyte development (Shen et al. 2005, Rama Raju et al. 2007). In our study, IL-ZP area and thickness were increased in positively activated oocytes in accordance with reports by Shen and coworkers (Shen et al. 2005) and Rama Raju and coworkers (Rama Raju et al. 2007).

The equine oocyte is characterized by the presence of abundant cytoplasmic granulation (Caamaño et al. 2013), which often prevents meiotic spindle display. Therefore, unlike in human and bovine oocyte analyses, the investigation of equine gametes could be based solely on the evaluation of the ZP.

Successfully activated bovine oocytes showed lower retardance values compared with oocytes that failed cleavage (Koester et al. 2011). Similarly, fertilized oocytes that cleaved exhibited lower birefringence/ retardance values compared to their counterparts that failed fertilization. Those data are in contrast with our findings, even if our results were not statistically significant.

Polscopic analysis of birefringent oocyte structures revealed predictive values for the quality and competence of equine, bovine and human oocytes, which are related to the results of parthenogenetic activation. These findings suggest that greater use of PLM in veterinary medicine could improve the selection of good-quality oocytes subjected to further in vitro fertilization techniques. In particular, the use of PLM in assisted reproduction in equines could be helpful to obtain greater success rates in ICSI cycles; those recorded to date have an average percentage of 
blastocyst formation of $5-15 \%$ (Choi et al. 2004a,b). By only selecting gametes with the best morphological characteristics, which are related to ZP birefringence, to be submitted for further fertilization techniques, better fertilization results could be expected.

In conclusion, our paper proves that (a) after IVM, equine oocytes can be evaluated using classical microscopy for the observation of morphological parameters as well as using PLM; (b) PLM is able to detect the differences between mature and immature oocytes and between mature oocytes that undergo activation and mature oocytes that do not activate; (c) IVM of equine oocytes causes changes in the inner layer structure of the ZP and (d) parthenogenic activation generates additional changes in the ZP structure.

Then, on a purely speculative level, it can be assumed that these variations, detectable by PLM, could be used to predict the ability of equine oocytes to be activated and that the predictive capacity of PLM could be higher than that of morphological evaluation obtained using a CLM, but more studies are required to prove it.

\section{Declaration of interest}

The authors declare that there is no conflict of interest that could be perceived as prejudicing the impartiality of the research reported.

\section{Funding}

This research did not receive any specific grant from any funding agency in the public, commercial or not-for-profit sector.

\section{References}

Alm H \& Hinrichs K 1996 Effect of cycloheximide on nuclear maturation of horse oocytes and its relation to initial cumulus morphology. Journal of Reproduction and Fertility 107 215-220. (doi:10.1530/jrf.0.1070215)

Caamaño JN, Muñoz M, Diez C \& Gómez E 2010 Polarized light microscopy in mammalian oocytes. Reproduction in Domestic Animals 45 49-56. (doi:10.1111/j.1439-0531.2010.01621.x)

Caamaño JN, Díez C, Trigal B, Muñoz M, Morató R, Martín D, Carrocera S, Mogas T \& Gómez E 2013 Assessment of meiotic spindle configuration and post-warming bovine oocyte viability using polarized light microscopy. Reproduction in Domestic Animals 48 470-476. (doi:10.1111/rda.12111)

Carneiro GF, Lorenzo PL, Pimentel C, Pegoraro L, Bertolini M, Ball BA, Anderson GB \& Liu IK 2001 Influence of insuline-like growth factor-I and its interaction with gonadotropins, estradiol, and fetal calf serum on in vitro maturation and parthenogenic development in equine oocytes. Biology of Reproduction 65 899-905. (doi:10.1095/biolreprod65.3.899)

Carneiro GF, Liu IK, Hyde D, Anderson GB, Lorenzo PL \& Ball PA 2002 Quantification and distribution of equine oocyte cortical granules during meiotic maturation and after activation. Molecular Reproduction and Development 63 451-458. (doi:10.1002/mrd.10198)

Choi YH, Love LB, Varner DD \& Hinrichs K 2004a Factors affecting developmental competence of equine oocytes after intracytoplasmic sperm injection. Reproduction 127 187-194. (doi:10.1530/rep.1.00087)

Choi YH, Roasa LM, Love CC, Varner DD, Brinsko SP \& Hinrichs K $2004 b$ Blastocyst formation rates in vivo and in vitro of in vitro-matured equine oocytes fertilized by intracytoplasmic sperm injection. Biology of Reproduction 70 1231-1238. (doi:10.1095/biolreprod.103.023903)

Cohen Y, Malcov M, Shwartz T, Mey-Raz N, Carmon A, Cohen T, Lessing JB, Amit A \& Azem F 2004 Spindle imaging: a new marker for optimal timing of ICSI? Human Reproduction 19 649-654. (doi:10.1093/ humrep/deh113)

Cooke S, Tyler JP \& Driscoll GL 2003 Meiotic spindle location and identification and its effect on embryonic cleavage plane and early development. Human Reproduction 18 2397-2405. (doi:10.1093/ humrep/deg447)

Dell'Aquila ME, Cho YS, Minoia P, Traina V, Lacalandra GM \& Maritato F 1997a Effect of follicular fluid supplementation of in-vitro maturation medium on the fertilization and development of equine oocyte after in vitro fertilization or intracytoplasmic sperm injection Human Reproduction 12 2766-2772. (doi:10.1093/humrep/12.12.2766)

Dell'Aquila ME, Cho YS, Minoia P, Traina V, Fusco S, Lacalandra GM \& Maritato F $1997 b$ Intracytoplasmic sperm injection (ICSI) versus conventional IVF on abattoir-derived and in vitro matured equine oocytes. Theriogenology 47 1139-1156. (doi:10.1016/S0093-691X(97)00095-2)

Ebner T, Moser M, Shebl O, Sommergruber M, Yaman C \& Tews G 2008 Blood clots in the cumulus oocyte-complex predict poor oocyte quality and post-fertilization development. Reproductive Biomedicine Online 16 801-807. (doi:10.1016/S1472-6483(10)60145-9)

Fernandes CB, Devito LG, Martins LR, Blanco ID, de Lima Neto JF, Tsuribe PM, Gonçalves CG \& da Cruz Landim-Alvarenga F 2014 Artificial activation of bovine and equine oocytes with cycloheximide, roscovitine, strontium, or 6-dimethylaminopurine in low or high calcium concentrations. Zygote 22 387-394. (doi:10.1017/ S0967199412000627)

Franz LC, Choi YH, Squires EL \& Hinrichs K 2003 Effects of roscovitine on maintenance of the germinal vesicle in horse oocytes, subsequent nuclear maturation, and cleavage rates after intracytoplasmic sperm injection. Reproduction 125 693-700. (doi:10.1530/rep.0.1250693)

Galli C \& Lazzari G 2001 In vitro and in vivo culture in the sheep oviduct of equine embryos obtained by IVM and ICSI. In Proceedings of the Second Meeting of the European Equine Gamete Group, pp 55-56. Eds TAE Stout \& JF Wade. Loosdrecht, The Netherlands Newmarket: R\&W Publications Abstract.

Galli C, Crotti G, Turini P, Duchi R, Mari G, Zavaglia G, Duchamp G, Daels P \& Lazzari G 2002 Frozen-thawed embryos produced by ovum pickup of immature oocyte and ICSI are capable to establish pregnancies in the horse. Theriogenology 58 705-708. (doi:10.1016/S0093691X(02)00771-9)

Galli C, Colleoni S, Duchi R, Lagutina I \& Lazzari G 2007 Developmental competence of equine oocytes and embryos obtained by in vitro procedures ranging from in vitro maturation and ICSI to embryo culture, cryopreservation, and somatic cell transfer. Animal Reproduction Science 98 39-55. (doi:10.1016/j.anireprosci.2006.10.011)

Gambini A, Jarazo J, Karlanian F, De Stéfano A \& Salamone DF 2014 Effect of collection-maturation interval time and pregnancy status of donor mares on oocyte developmental competence in horse cloning. Journal of Animal Science 92 561-567. (doi:10.2527/jas.2013-7026)

Held E, Mertens EM, Mohammadi-Sangcheshmeh A, Salilew-Wondim D, Besenfelder U, Havlicek V, Herrler A, Tesfaye D, Schellander K \& Hölker M 2012 Zona pellucida birefringence correlates with developmental capacity of bovine oocytes classified by maturational environment, COC morphology and G6PDH activity. Reproduction, Fertility and Development 24 568-579. (doi:10.1071/RD11112)

Hinrichs K 2010 The equine oocyte: factors affecting meiotic and developmental competence. Molecular Reproduction and Development 77 651-661. (doi:10.1002/mrd.21186)

Hinrichs K 2011 Immature oocyte collection and maturation. In Equine Reproduction, edn 2, p 2934. Eds AO McKinnon, EL Squires, WE Vaala \& DD Varner. New Delhi, India: Wiley-Blackwell.

Hinrichs K \& Schmindt AL 2000 Meiotic competence in horse oocyte: interactions among chromatin configuration, follicle size, cumulus morphology and season. Biology of Reproduction 62 1402-1408. (doi:10.1095/biolreprod62.5.1402)

Hinrichs K \& Williams KA 1997 Relationships among oocyte-cumulus morphology, follicular atresia, initial chromatin configuration, and oocyte meiotic competence in the horse. Biology of Reproduction $\mathbf{5 7}$ 377-384. (doi:10.1095/biolreprod57.2.377) 
Hinrichs K, Schmidt AL, Friedman PP, Selgrath JP \& Martin MG 1993 In vitro maturation of horse oocytes: characterization of chromatin configuration using fluorescence microscopy. Biology of Reproduction 48 363-370. (doi:10.1095/biolreprod48.2.363)

Hinrichs K, Schmidt AL \& Selgrath JP 1995 Activation of horse oocytes. In Equine Reproduction VI, 1, pp 319-324. Eds DC Sharp \& FW Bazer. Madison, WI: Society for the Study of Reproduction.

Hinrichs K, Choi YH, Love LB, Love CC, Varner DD \& Ingram LA 2002 Effects of holding time and media on meiotic and developmental competence of horse oocytes. Theriogenology 58 675-678. (doi:10.1016/S0093691X(02)00802-6)

Hinrichs K, Choi YH, Love LB, Varner DD, Love CC \& Walckenaer BE 2005 Chromatin configuration within the germinal vesicle of horse oocytes: changes post mortem and relationship to meiotic and developmental competence. Biology of Reproduction 72 1142-1150. (doi:10.1095/ biolreprod.104.036012)

Keefe D, Tran P, Pellegrini C \& Oldenbourg R 1997 Polarized light microscopy and digital image processing identify a multilaminar structure of the hamster zona pellucida. Human Reproduction 12 1250-1252. (doi:10.1093/humrep/12.6.1250)

Keefe D, Liu L, Wang W \& Silva C 2003 Imaging meiotic spindles by polarization light microscopy: principles and applications to IVF. Reproductive Biomedicine Online 7 24-29. (doi:10.1016/S14726483(10)61724-5)

Koester M, Mohammadi-Sangcheshmeh A, Montag M, Rings F, Schimming T, Tesfaye D, Schellander K \& Hoelker M 2011 Evaluation of bovine zona pellucida characteristics in polarized light as a prognostic marker for embryonic developmental potential. Reproduction 141 779-787. (doi:10.1530/REP-10-0471)

Kilani SS, Cooke S, Kan AK \& Chapman MG 2006 Do age and extended culture affect the architecture of the zona pellucida of human oocytes and embryos? Zygote 14 39-44. (doi:10.1017/S0967199406003625)

Lagutina I, Lazzari G, Duchi R, Colleoni S, Ponderato N, Turini P, Crotti G \& Galli C 2005 Somatic cell nuclear transfer in horses: effect of oocyte morphology, embryo reconstruction method and donor cell type. Reproduction 130 559-567. (doi:10.1530/rep.1.00772)

Liu L, Ju J \& Yang X 1998 Differential inactivation of maturation-promoting factor and mitogen-activated protein kinase following parthenogenic activation of bovine oocytes. Biology of Reproduction 59 537-545. (doi:10.1095/biolreprod59.3.537)

Madaschi C, Aoki T, Paes de Almeida Ferreira Braga D, de Cássia Sávio Figueira R, Semião Francisco L, laconelli A \& Borges E 2009 Zona pellucida birifrangence score and meiotic spindle visualization in relation to embryo development and ICSI outcomes. Reproductiove Biomedicine Online 18 681-686. (doi:10.1016/S1472-6483(10)60014-4)

Mohammadi-Sangcheshmeh A, Held E, Rings F, Ghanem N, SalilewWondim D, Tesfaye D, Sieme H, Schellander K \& Hoelker M 2014 Developmental competence of equine oocytes: impacts of zona pellucida birefringence and maternally derived transcript expression. Reproduction, Fertility and Development 26 441-452. (doi:10.1071/ RD12303)

Molinari E, Evangelista F, Racca C, Cagnazzo C \& Revelli A 2012 Polarized light microscopy-detectable structures of human oocytes and embryos are related to the likelihood of conception in IVF. Journal of Assisted Reproduction and Genetics 29 1117-1122. (doi:10.1007/s10815-0129840-9)

Montag M \& van der Ven H 2008 Symposium: innovative techniques in human embryo viability assessment. Oocyte assessment and embryo viability prediction: birefringence imaging. Reproductiove Biomedicine Online 17 454-460. (doi:10.1016/S1472-6483(10)60231-3)

Moon JH, Hyun CS, Lee SW, Son WY, Yoon SH \& Lim JH 2003 Visualization of the metaphase II meiotic spindle in living human oocytes using the PolScope enables the prediction of embryonic developmental competence after ICSI. Human Reproduction 18 817-820. (doi:10.1093/ humrep/deg165)
Ongeri EM, Bormann CL, Butler RE, Melican D, Gavin WG, Echelard Y, Krisher RL \& Behboodi E 2001 Development of goat embryos after in vitro fertilization and parthenogenetic activation by different methods. Theriogenology 55 1933-1945. (doi:10.1016/S0093-691X(01)00534-9)

Pedersen HG, Watson ED \& Telfer EE 2004 Effect of ovary holding temperature and time on equine granulosa cell apoptosis, oocyte chromatin configuration and cumulus morphology. Theriogenology 62 468-480. (doi:10.1016/j.theriogenology.2003.10.006)

Pelletier C, Keefe DL \& Trimarchi JR 2004 Noninvasive polarized light microscopy quantitatively distinguishes the multilaminar structure of the zona pellucida of living human eggs and embryos. Fertility and Sterility 81 850-856. (doi:10.1016/j.fertnstert.2003.09.033)

Pereira GR, Lorenzo PL, Carneiro GF, Bilodeau-Goeseels S, Kastelic JP, Esteller-Vico A, Lopez-Bejar M \& Liu IK 2014 Selection of developmentally competent immature equine oocytes with brilliant cresyl blue stain prior to in vitro maturation with equine growth hormone. Zygote 22 500-504. (doi:10.1017/S096719941200072X)

Rama Raju GA, Prakash GJ, Krishna KM \& Madan K 2007 Meiotic spindle and zona pellucida characteristics as predictors of embryonic development: a preliminary study using PolScope imaging. Reproductive Biomedicine Online 14 166-174. (doi:10.1016/S1472-6483(10)60784-5)

Rienzi L, Martinez F, Ubaldi F, Minasi MG, lacobelli M, Tesarik J \& Greco E 2004 Polscope analysis of meiotic spindle changes in living metaphase II oocytes during the freezing and thawing procedures. Human Reproduction 19 655-659. (doi:10.1093/humrep/deh101)

Shen Y, Stalf T, Mehnert C, Eichenlaub U \& Tinneberg HR 2005 High magnitude of light retardation by the zona pellucida is associated with conception cycle. Human Reproduction 20 1596-1606. (doi:10.1093/ humrep/deh811)

Siddiqui MA, Gastal EL, Ju JC, Gastal MO, Beg MA \& Ginther OJ 2009 Nuclear configuration, spindle morphology and cytoskeletal organization of in vivo maturing horse oocytes. Reproduction in Domestic Animals 44 435-440. (doi:10.1111/j.1439-0531.2008.01105.x)

Tharasanit T, Colleoni S, Lazzari G, Colenbrander B, Galli C \& Stout TA 2006 Effect of cumulus morphology and maturation stage on the cryopreservability of equine oocytes. Reproduction 132 759-769. (doi:10.1530/rep.1.01156)

Tremoleda JL, Stout TA, Lagutina I, Lazzari G, Bevers MM, Colenbrander B \& Galli C 2003 Effects of in vitro production on horse embryo morphology, cytoskeletal characteristics, and blastocyst capsule formation. Biology of Reproduction 69 1895-1906. (doi:10.1095/biolreprod.103.018515)

Trimarchi JR, Karin RA \& Keefe D 2004 Average spindle retardance observed using the polscope predicts cell number in day 3 embryos. Fertility and Sterility 82 S268. (doi:10.1016/j.fertnstert.2004.07.716)

Wang WH, Meng L, Hackett RJ \& Keefe DL 2001a Developmental ability of human oocytes with or without birefringent spindles imaged by PolScope before insemination. Human Reproduction 16 1464-1468. (doi:10.1093/humrep/16.7.1464)

Wang WH, Meng L, Hackett RJ, Odenbourg R \& Keefe DL 2001b The spindle observation and its relationship with fertilization after intracytoplasmic sperm injection in living human oocytes. Fertility and Sterility 75 348-353. (doi:10.1016/S0015-0282(00)01692-7)

Willis P, Caudle AB \& Fayrer-Hosken A 1991 Equine oocyte in vitro maturation: influence of sera, time and hormones. Molecular Reproduction and Development 30 360-368. (doi:10.1002/mrd.1080300411)

Yeste M, Jones C, Amdani SN, Patel S \& Coward K 2016 Oocyte activation deficiency: a role for an oocyte contribution? Human Reproduction Update 22 23-47. (doi:10.1093/humupd/dmv040)

Received 16 January 2017

First decision 19 February 2017

Revised manuscript received 3 March 2017

Accepted 9 March 2017 